\section{New link between myosin VI and prostate cancer}

Dunn and colleagues have analyzed global gene expression in normal prostate epithelium and cancerous acini, and discovered unexpectedly consistent overexpression of myosin VI mRNA in clinical prostate specimens. The group constructed tissue microarrays of prostate tissue specimens from 240 patients, and used immunohistochemical analysis and functional studies of human prostate cancer cell lines to investigate the role of myosin $\mathrm{VI}$ in human prostate cancer.

Cancer samples showed 3.7-fold higher expression of myosin VI mRNA when compared with normal samples. Weighted geneexpression analysis also showed that myosin VI was one of the most highly overexpressed genes in cancerous human prostate tissue, compared with normal tissue $\left(P<10^{-10}\right)$. Prostate cancers with Gleason scores of 6-7 demonstrated the most consistent overexpression of myosin VI, although high-grade, histologically aggressive cancers (Gleason score 8-10) also showed marked overexpression of myo$\sin \mathrm{VI}$, albeit to a lesser extent than cancers of Gleason score 6-7 (both comparisons with normal tissues). Examination of human prostate cancer cell lines also revealed a general pattern of decreased cancer-specific myosin VI expression in high-grade cancers.

These results reveal a novel connection between myosin $\mathrm{VI}$ and human prostate cancer. Further studies are required to characterize the unique structural and functional properties of myosin VI fully, and to investigate its role in the development of prostate cancer.

Original article Dunn TA et al. (2006) A novel role of myosin VI in human prostate cancer. Am J Pathol 169: 1843-1854

\section{Nerve-sparing cystectomy helps preserve sexual and urinary function}

Although radical cystectomy is an effective treatment for bladder cancer, it can have negative effects on urinary and sexual function. Lane et al. have performed nerve-sparing and reproductive-organ-sparing laparoscopic radical cystectomy (LRC) on five patients, and in a recent article in Urology describe their technique and short-term results.
Four male patients with muscle-invasive bladder cancer underwent nerve-sparing LRC. The technique involved identifying and completely releasing the neurovascular bundle before division of the urethra, which minimized damage to the neurovascular bundle along the prostatic apex. One woman with muscle-invasive bladder cancer also underwent the procedure. The uterus, fallopian tubes, ovaries, and vagina were preserved, the endopelvic fascia was left intact, and manipulation of the urethra was minimal. In all procedures, transection of the lateral vascular pedicle and posterior dissection was carried out as close as possible to the bladder.

The median total operative time was $10 \mathrm{~h}$, median blood loss was $400 \mathrm{ml}$, median hospital stay was 5 days, and median follow-up was 30 months. No patients showed signs of recurrence. At 12 months, nocturnal continence was preserved in all patients, daytime continence was preserved in three patients, and the female patient and two of the male patients retained sexual function.

Although only $50 \%$ of the male patients retained sexual function, this result is superior to the results observed with non-nerve-sparing radical cystectomy (3-13\%). Lane et al. recommend that nerve-sparing LRC be performed in cases of low-volume, organ-confined bladder cancer in younger patients.

Original article Lane BR et al. (2006) Nerve-sparing laparoscopic radical cystectomy: technique and initial outcomes. Urology 68: 778-783

\section{Vardenafil has an extended, $8 \mathrm{~h}$ duration of action}

For many couples sexual activity is unplanned, so phosphodiesterase type 5 inhibitor therapy that allows a wide time-frame for achieving successful intercourse would give couples more freedom to engage in sexual activity than existing regimes permit. Vardenafil has previously been demonstrated to be effective and well tolerated, and to have a rapid onset of action in men with erectile dysfunction. In this prospective study, Porst et al. have now shown that vardenafil remains effective $8 \mathrm{~h}$ after administration.

Their international, multicenter, randomized, double-blind, placebo-controlled study included 383 men who had erectile dysfunction of 